\title{
Multimorbidity and Opioid Prescribing in Hospitalized Older Adults
}

\author{
Sarah Schear, MS, ${ }^{1}$ Kanan Patel, MBBS, MPH, ${ }^{1}$ Lisa X. Deng, BS, ${ }^{1}$ Christine Miaskowski, PhD, RN, ${ }^{2}$ \\ Ingrid Maravilla, BA, ${ }^{1}$ Sarah K. Garrigues, BA, Nicole Thompson, BA, ${ }^{1}$ \\ Andrew D. Auerbach, MD, MPH, and Christine S. Ritchie, MD, MSPH ${ }^{1}$
}

\begin{abstract}
Background: Multimorbidity and pain are both common among older adults, yet pain treatment strategies for older patients with multimorbidity have not been well characterized.

Objectives: To assess the prevalence and relationship between multimorbidity and opioid prescribing in hospitalized older medical patients with pain.

Methods: We collected demographic, morbidity, pain, and analgesic treatment data through structured review of the electronic medical records of a consecutive sample of 238 medical patients, aged $\geq 65$ years admitted between November 2014 and May 2015 with moderate-to-severe pain by numerical pain rating scale (range 4-10). We used the Cumulative Illness Rating Scale for Geriatrics (CIRS-G) to assess multimorbidity and cumulative illness burden. We examined the relationship between morbidity measures and opioid prescribing at hospital discharge using multivariate regression analysis.

Results: The mean age was $75 \pm 8$ years, $57 \%$ were female and $50 \%$ were non-White. Mean CIRS-G total score was $17 \pm 6$, indicating high cumulative illness burden. Ninety-nine percent of patients had multimorbidity, defined as moderate-to-extremely severe morbidity in $\geq 2$ organ systems. Sixty percent of patients received an opioid prescription at discharge. In multivariate analyses adjusted for age, race, and gender, patients with a discharge opioid prescription were significantly more likely to have higher cumulative illness burden and chronic pain.

Conclusion: Among older medical inpatients, multimorbidity was nearly universal, and patients with higher cumulative illness burden were more likely to receive a discharge opioid prescription. More studies of benefits and harms of analgesic treatments in older adults with multimorbidity are needed to guide clinical practice.
\end{abstract}

Keywords: hospitalization; illness burden; multimorbidity; older adults; opioids

\section{Introduction}

$\mathbf{M}$ ULTIMORBIDITY, defined as the presence of $\geq 2$ comorbid illnesses, commonly afflicts community-dwelling older adults, involving about half of people aged 65-69 years and more than three quarters $>85$ years of age. ${ }^{1-3}$ Multimorbidity in older adults contributes to reduced functional status and lower quality of life, and increased inpatient days, rehospitalization, and mortality.

In community-dwelling older adults with multimorbidity, pain is among the most reported symptoms. ${ }^{7}$ For older adults living with pain, ${ }^{8-11}$ pharmacotherapy is the most common pain treatment they recieved. ${ }^{12}$ However, the pres- ence and severity of comorbid conditions affect analgesic safety in this population. For example, nonsteroidal antiinflammatory drugs (NSAIDs) incur greater risk in older adults with age- or disease-related declines in renal function. ${ }^{13}$ Studies of opioid-related adverse events in surgical inpatients suggest an association between older age, comorbidity severity, and opioid-related adverse events. ${ }^{14-16}$ Nevertheless, multimorbidity remains an understudied and under-recognized reality in pain treatment guidelines. ${ }^{2}$ Despite the high prevalence of pain and multimorbidity in older adults, little is known about patterns of analgesic treatment for these patients, particularly in the medical inpatient setting. ${ }^{17,18}$

\footnotetext{
${ }^{1}$ Division of Geriatrics, Department of Medicine, University of California San Francisco, San Francisco, California.

${ }^{2}$ Department of Physiological Nursing, School of Nursing, University of California San Francisco, San Francisco, California

${ }^{3}$ Division of Hospital Medicine, Department of Medicine, University of California San Francisco, San Francisco, California.

Accepted September 24, 2019.
} 
We undertook this study to describe the prevalence of multimorbidity and its relationship with opioid prescribing in hospitalized older adults with pain. We focused on opioid prescribing due to heightened national attention on opioid prescribing and the Centers for Disease Control and Prevention's (CDC) recommendation to prescribe opioids with caution in older adults. ${ }^{19}$ We hypothesized that patients with an opioid prescription at hospital discharge would have greater levels of morbidity than patients discharged without an opioid prescription.

\section{Methods}

\section{Population and setting}

We collected data from medical records of patients aged $\geq 65$ years admitted to medicine services at an urban academic medical center from November 2014 to May 2015. The study methods and sampling criteria (Fig. 1) are reported elsewhere. ${ }^{20}$ We included patients in the study if they had moderate-to-severe pain (pain score of $\geq 4$ on a numerical pain rating scale [NPRS; 11 points scale] or $\geq 1$ on the Checklist of Non-Verbal Pain Indicators $\left.[\mathrm{CNPI}]^{21-23}\right)$ at admission $(n=248)$ and did not die during hospitalization $(n=10)$.

Trained research personnel collected data by structured chart abstraction from the electronic medical record (EMR). Ethical approval was obtained from the local institutional review board.

\section{Independent measures}

Demographic data included age at hospitalization, gender, race, ethnicity, marital status, insurance type, language preference, hospitalization length, and admission and discharge locations.

Pain. We abstracted admission and discharge pain scores-defined as NPRS, CNPI, and Verbal Descriptor
Scale (VDS) ${ }^{24}$ pain scores documented within 24 hours of admission and 48 hours before discharge, respectively. To harmonize the three pain scales, all CNPI and VDS pain scores were converted to corresponding NPRS scores using a previously described algorithm. ${ }^{20}$

Measures of morbidity. Multimorbidity has several features: (1) severity of the individual comorbid condition, (2) sum of high-severity comorbid conditions, and (3) combined severity of all comorbidity conditions resulting in overall illness burden. We chose the Cumulative Illness Rating Scale for Geriatrics (CIRS-G) to assess these three multimorbidity characteristics in our cohort. ${ }^{6}$ The scale assesses 14 body systems rated on a 5-point scale from 0 (no illness) to 4 (extremely severe, intractable illness). Although CIRS-G scores range from 0 to 56 , very high scores are incompatible with life as they indicate multiorgan failure. ${ }^{5}$ The scale has good inter-rater reliability and validity in capturing overall illness burden for both inpatient and outpatient older adults. $^{6,25}$

Trained clinical personnel completed the CIRS-G scoring. The 1991 CIRS-G manual ${ }^{26}$ was the main scoring reference due to its extensive validation ${ }^{6,27}$; the 2008 CIRS manual $^{5}$ was used to clarify ambiguous scenarios. Scorers conducted a structured assessment of each patient's admission and discharge notes. Scorers also reviewed the emergency department note, the last preceding primary care note, laboratory results, or keyword search results when needed. To ensure inter-rater reliability, the first 100 charts were double scored. All discrepancies were resolved in consultation with the senior author (C.S.R.).

We used various measures from the CIRS-G to indicate the three different aspects of multimorbidity. We used the $C I R S-G$ score for each organ system to describe individual systemspecific illness severity. We summed the number of organ systems with a score of $\geq 2$ to assess multimorbidity severity.

3238 patients Age $\geq 65$ years admitted between 11/28/2014 - 5/28/2015

Excluded:

- 1691 admitted to other services

- 82 admitted <24 hours

1465 patients admitted to medicine services

Excluded:

- 179 with missing/inadequate pain data

- 19 received surgery during hospitalization

- 10 deceased during hospitalization

- 938 no pain

- 81 mild pain

Final study sample: 238 patients with

moderate-to-severe pain at admission

(Numerical Pain Rating Scale 4-10) and

alive through discharge

FIG. 1. Sampling flow chart of study participants. 
We used the CIRS-G total score to characterize cumulative illness burden. We chose the term "burden" because it captures the overall impact of each comorbidity in a person, taking severity into account. ${ }^{28}$

\section{Outcome measures}

Our major outcome of interest was opioid analgesic prescribing at hospital discharge. This variable included both new prescriptions resulting from the hospitalization and continued prescriptions to patients already prescribed an opioid before admission. Our secondary outcome assessed the receipt of higher dose opioids in the 24 hours before discharge. We converted all opioid doses to their morphine equivalent daily dose (MEDD), and we defined a high dose as MEDD $\geq 50 \mathrm{mg}$ based on the 2016 CDC guidelines. ${ }^{19}$

We also collected data on other analgesics (e.g., acetaminophen, NSAIDs, and adjuvants) prescribed at hospital discharge and on nonpharmacologic pain treatments (e.g., hot and cold application, repositioning) documented during hospitalization.

\section{Statistical analysis}

We compared differences in patients' characteristics with and without an opioid prescription at discharge using $\chi^{2}$ tests for categorical variables and Student's $t$-tests for continuous variables. Multivariate logistic regression was used to estimate the odds ratio (OR) for characteristics associated with opioid prescription at discharge, and with high opioid dose. These models considered covariates of age, gender, race, and history of chronic pain. Analyses were conducted using SAS version 9.4 (SAS Institute, Inc., Cary, NC).

\section{Results}

Older adults admitted with moderate-to-severe pain $(n=238)$ had a mean age of $74.9 \pm 8.2$ years, $56.7 \%$ were female, $49.6 \%$ were non-White, and $39.9 \%$ had a history of chronic pain (Table 1). Patients had a mean CIRS-G total score of 17.2 \pm 5.5 , indicating high cumulative illness burden (Table 2).

\section{Opioid prescriptions}

Sixty percent $(n=142)$ of patients received an opioid prescription at discharge (Table 1). Among patients receiving discharge opioids, $75.4 \%$ already had an opioid prescription before admission. In the bivariate analysis, patients were more likely to receive discharge opioid prescriptions if they had a chronic pain diagnosis $(49.3 \%$ vs. $26.0 \%, p<0.001)$, cancer diagnosis ( $44.4 \%$ vs. $22.9 \%, p<0.001)$, English language preference $(77.5 \%$ vs. $62.5 \%, p=0.012)$, and higher discharge pain scores (mean of $2.4 \pm 2.7$ vs. $1.4 \pm 2.5$, $p=0.002$, Table 1). Among patients without a preadmission opioid prescription $(n=118)$, the incident prescription rate was $14.7 \%$.

Notably, opioid prescriptions were associated with prescription of other pharmacologic and nonpharmacologic pain treatments. Compared with patients not receiving opioids, those receiving discharge opioids were more likely to receive acetaminophen $(69.7 \%$ vs. $33.3 \%, p<0.001)$ and adjuvant analgesics $(34.5 \%$ vs. $21.9 \%, p=0.036)$ prescription at discharge and nonpharmacologic treatment order $(56.3 \%$ vs. $35.4 \%, p=0.002$ ) during their hospitalization.

Among patients with discharge opioids $(n=142), 35.2 \%$ received a high dose (MEDD $\geq 50 \mathrm{mg}$ ) of opioids within 24 hours before discharge (Table 3 ). Compared with those

Table 1. Characteristics of Patients Admitted to Medicine Service with Moderate-to-Severe Pain, Overall and by Opioid Prescription at Discharge

\begin{tabular}{|c|c|c|c|c|}
\hline \multirow[b]{2}{*}{ Characteristics, $\mathrm{n}(\%)$ or mean \pm standard deviation } & \multirow[b]{2}{*}{ Overall $(\mathrm{n}=238)$} & \multicolumn{2}{|c|}{ Discharge opioid prescription } & \multirow[b]{2}{*}{$\mathrm{p}$} \\
\hline & & No $(\mathrm{n}=96)$ & Yes $(\mathrm{n}=142)$ & \\
\hline Age in years & $74.9 \pm 8.2$ & $75.5 \pm 8.1$ & $74.4 \pm 8.3$ & 0.30 \\
\hline Female & $135(56.7)$ & $51(53.1)$ & $84(59.2)$ & 0.36 \\
\hline \multicolumn{5}{|l|}{ Race and ethnicity } \\
\hline White & $120(50.4)$ & $45(46.9)$ & $75(52.8)$ & 0.57 \\
\hline Asian & $52(21.9)$ & $24(25.0)$ & $28(19.7)$ & \\
\hline Other & $66(27.7)$ & $27(28.1)$ & $39(27.5)$ & \\
\hline Hispanic & $26(10.9)$ & $15(15.6)$ & $11(7.7)$ & 0.06 \\
\hline \multicolumn{5}{|l|}{ Language and residence } \\
\hline Preferred language of English & $170(71.4)$ & $60(62.5)$ & $110(77.5)$ & 0.012 \\
\hline Admission from community & $216(90.8)$ & $87(90.6)$ & $129(90.8)$ & 0.95 \\
\hline Discharge to community & $147(61.8)$ & $61(63.5)$ & $86(60.6)$ & 0.64 \\
\hline \multicolumn{5}{|l|}{ Conditions in medical history } \\
\hline Chronic pain & $95(39.9)$ & $25(26.0)$ & $70(49.3)$ & $<0.001$ \\
\hline Depression & $61(25.6)$ & $20(20.8)$ & $41(28.9)$ & 0.16 \\
\hline Cancer & $85(35.7)$ & $22(22.9)$ & $63(44.4)$ & $<0.001$ \\
\hline \multicolumn{5}{|l|}{ Admission characteristics } \\
\hline Admitted with existing opioid prescription & $120(50.4)$ & $13(13.5)$ & $107(75.4)$ & $<0.001$ \\
\hline Opioid prescribed during admission & $189(79.4)$ & $55(29.1)$ & $134(70.9)$ & $<0.001$ \\
\hline Hospital length of stay & $5.7 \pm 6.5$ & $4.3 \pm 4.0$ & $6.6 \pm 7.6$ & 0.002 \\
\hline Admission pain score & $6.3 \pm 1.8$ & $6.1 \pm 1.8$ & $6.5 \pm 1.9$ & 0.14 \\
\hline Discharge pain score & $2.0 \pm 2.7$ & $1.4 \pm 2.5$ & $2.4 \pm 2.7$ & 0.002 \\
\hline
\end{tabular}


Table 2. Measures of Morbidity in Patients Admitted to Medicine Service with Moderate-to-Severe Pain, Overall and by Opioid Prescription at Discharge

\begin{tabular}{|c|c|c|c|c|}
\hline \multirow[b]{2}{*}{ Characteristics, $\mathrm{n}(\%)$ or mean \pm standard deviation } & \multirow[b]{2}{*}{ Overall $(\mathrm{n}=238)$} & \multicolumn{2}{|c|}{ Discharge opioid prescription } & \multirow[b]{2}{*}{$\mathrm{p}$} \\
\hline & & No $(\mathrm{n}=96)$ & $\operatorname{Yes}(\mathrm{n}=142)$ & \\
\hline Cumulative illness burden (CIRS-G total score) & $17.2 \pm 5.5$ & $15.8 \pm 5.6$ & $18.1 \pm 5.3$ & 0.002 \\
\hline \multicolumn{5}{|c|}{ System-specific illness severity (CIRS-G score by organ system, 0-4) } \\
\hline Heart & $1.36 \pm 1.4$ & $1.40 \pm 1.3$ & $1.33 \pm 1.4$ & 0.72 \\
\hline Vascular & $1.92 \pm 1.2$ & $1.74 \pm 1.1$ & $2.04 \pm 1.2$ & 0.049 \\
\hline Hematologic & $1.45 \pm 1.2$ & $1.24 \pm 1.2$ & $1.59 \pm 1.2$ & 0.027 \\
\hline Respiratory & $1.50 \pm 1.5$ & $1.25 \pm 1.4$ & $1.66 \pm 1.6$ & 0.037 \\
\hline Eyes, ears, nose, and throat and larynx & $0.82 \pm 1.0$ & $0.73 \pm 1.0$ & $0.87 \pm 1.0$ & 0.27 \\
\hline Upper gastrointestinal & $1.24 \pm 1.2$ & $1.13 \pm 1.2$ & $1.31 \pm 1.2$ & 0.25 \\
\hline Lower gastrointestinal & $1.13 \pm 1.3$ & $1.13 \pm 1.2$ & $1.12 \pm 1.4$ & 0.93 \\
\hline Liver & $0.83 \pm 1.2$ & $0.95 \pm 1.3$ & $0.75 \pm 1.2$ & 0.21 \\
\hline Renal & $0.76 \pm 1.2$ & $0.68 \pm 1.1$ & $0.81 \pm 1.2$ & 0.39 \\
\hline Genitourinary & $0.96 \pm 1.1$ & $1.00 \pm 1.1$ & $0.93 \pm 1.1$ & 0.64 \\
\hline Musculoskeletal/integument & $1.81 \pm 1.2$ & $1.35 \pm 1.0$ & $2.11 \pm 1.2$ & $<0.001$ \\
\hline Neurologic & $0.95 \pm 1.3$ & $0.85 \pm 1.3$ & $1.01 \pm 1.3$ & 0.34 \\
\hline Endocrine/metabolic/breast & $0.99 \pm 1.1$ & $0.95 \pm 1.1$ & $1.02 \pm 1.2$ & 0.62 \\
\hline Psychiatric & $1.47 \pm 1.3$ & $1.42 \pm 1.3$ & $1.51 \pm 1.3$ & 0.57 \\
\hline \multicolumn{5}{|c|}{ Multimorbidity severity (number of organ systems with CIRS-G score of $\geq 2$ ) } \\
\hline Two or more systems & $235(98.7)$ & $93(96.9)$ & $142(100.0)$ & 0.07 \\
\hline Three or more systems & $226(95.0)$ & $88(91.7)$ & $138(97.2)$ & 0.07 \\
\hline Four or more systems & $198(83.2)$ & $73(76.0)$ & $125(88.0)$ & 0.015 \\
\hline
\end{tabular}

Each organ system scored $0-4$ for illness severity: $0=$ none, $1=$ mild, $2=$ moderate, $3=$ severe, $4=$ extremely severe.

CIRS-G, Cumulative Illness Rating Scale for Geriatrics.

receiving an MEDD $<50 \mathrm{mg}$, those who received a high dose were younger (mean age $71.7 \pm 7.4$ vs. $75.9 \pm 8.4$ years, $p=0.004)$ and were less likely to be discharged to the community $(48.0 \%$ vs. $67.4 \%, p=0.024)$. Patients were more likely to receive a high dose if they had a history of chronic pain $(64.0 \%$ vs. $41.3 \%, p=0.01)$, depression (42.0\% vs. $21.7 \%, p=0.011)$, or metastatic cancer $(36.0 \%$ vs. $15.2 \%$, $p=0.005$ ) and had higher discharge pain scores (mean of $3.4 \pm 2.7$ vs. $1.9 \pm 2.5, p=0.002)$.

\section{Multimorbidity and illness burden}

Multimorbidity was nearly universal in this cohort: $99 \%$ of patients had moderate-to-extremely severe morbidity in $\geq 2$ organ systems, $95 \%$ in $\geq 3$ systems, and $83 \%$ in $\geq 4$ systems (Table 2). The most common morbidities were vascular (affecting $72.7 \%$ ), musculoskeletal $(63.5 \%)$, psychiatric $(52.1 \%)$, and upper gastrointestinal (47.9\%) (data not shown). Although liver $(24.0 \%)$ and renal $(21.9 \%)$ systems had the lowest prevalence of morbidity, they still affected a large proportion of patients.

\section{Relationship between measures of morbidity and opioid prescription}

Cumulative illness burden was significantly higher in patients with discharge opioid prescriptions (CIRS-G total score $18.1 \pm 5.3$ vs. $15.8 \pm 5.6, p=0.002$, Table 2). Multimorbidity severity was also significantly higher in the opioidprescribed group: $88 \%$ had $\geq 4$ body systems with high levels of illness severity compared with $76 \%$ in those without a prescription $(p=0.015)$. Patients with discharge opioid prescriptions had higher average scores for illness severity in vascular, hematopoietic, respiratory, and musculoskeletal systems (Table 2). Patients with a high opioid dose had significantly higher cumulative illness burden (CIRS-G total score $19.6 \pm 5.0$ vs. $17.2 \pm 5.3, p=0.01$, Table 3 ).

In regression analysis, the odds of receiving discharge opioids was 1.6 times greater among individuals with chronic pain (OR 2.59, 95\% confidence interval [CI] 1.45-4.61, $p=0.001)$ and was also higher in patients with higher cumulative illness burden (with each point increase in CIRS-G total score, OR 1.08, 95\% CI 1.03-1.14, $p=0.003$ ) after adjusting for age, gender, and race (Table 4$)$. This association persisted even if cancer patients $(n=85)$ were excluded from the analysis (OR 1.07, 95\% CI 1.01-1.14, $p=0.019)$. Furthermore, although the number of patients receiving a high MEDD dose was low $(n=50)$, the odds of receiving a higher dose was similarly elevated with chronic pain and with incremental increases in CIRS-G total score (data not shown).

\section{Discussion}

Few studies have described opioid prescribing to hospitalized older adults in the context of multimorbidity. $16,18,29,30$ This is the first study to assess the relationship between comorbidity severity and burden and opioid use in hospitalized older adults. In this cohort of older medical inpatients with moderate-to-severe pain, most patients entered (50.4\%) and left the hospital with an opioid prescription (59.7\%). Multimorbidity was nearly universal and cumulative illness burden was strikingly high. Discharge opioid prescriptions were associated with higher cumulative illness burden and a history of chronic pain. The association with illness burden persisted even after adjustment for age, gender, race, and chronic pain, and after exclusion of cancer patients. 
Table 3. Characteristics of Patients Admitted to Medicine Service with Moderate-to-Severe Pain by Opioid Dose Prescribed during 24 Hours before Discharge

\begin{tabular}{|c|c|c|c|}
\hline \multirow{2}{*}{$\begin{array}{l}\text { Characteristics, } \mathrm{n}(\%) \text { or } \\
\text { mean } \pm \text { standard deviation }\end{array}$} & \multicolumn{2}{|c|}{ Opioid Dose } & \multirow[b]{2}{*}{$\mathrm{p}$} \\
\hline & $M E D D<50(\mathrm{n}=92)$ & $M E D D \geq 50(\mathrm{n}=50)$ & \\
\hline MEDD dose, mg & $13.9 \pm 13.9$, range $0-45$ & $158.1 \pm 111.7$, range $60-448$ & $<0.001$ \\
\hline Age, years & $75.9 \pm 8.4$ & $71.7 \pm 7.4$ & 0.004 \\
\hline $65-74$ & $44(47.8)$ & $36(72.0)$ & 0.006 \\
\hline $75+$ & $48(52.2)$ & $14(28.0)$ & \\
\hline \multirow{2}{*}{\multicolumn{4}{|c|}{ Race and ethnicity }} \\
\hline & & & \\
\hline White & $43(46.7)$ & $32(64.0)$ & 0.14 \\
\hline Asian & $21(22.8)$ & $7(14.0)$ & \\
\hline Other & $28(30.4)$ & $11(22.0)$ & \\
\hline Hispanic & $7(7.6)$ & $4(8.0)$ & 0.93 \\
\hline \multicolumn{4}{|l|}{ Language and residence } \\
\hline Preferred language of English & 68 (73.9) & $42(84.0)$ & 0.17 \\
\hline Admission from community & 87 (94.6) & $42(84.0)$ & 0.037 \\
\hline Discharge to community & $62(67.4)$ & $24(48.0)$ & 0.024 \\
\hline \multicolumn{4}{|l|}{ Conditions in medical history } \\
\hline Chronic pain & $38(41.3)$ & $32(64.0)$ & 0.010 \\
\hline Depression & $20(21.7)$ & $21(42.0)$ & 0.011 \\
\hline Cancer & $36(39.1)$ & $27(54.0)$ & 0.09 \\
\hline Metastatic & $14(15.2)$ & $18(36.0)$ & 0.005 \\
\hline \multicolumn{4}{|l|}{ Admission characteristics } \\
\hline Hospital length of stay & $6.6 \pm 8.2$ & $6.6 \pm 6.4$ & 0.98 \\
\hline Admission pain score & $6.3 \pm 1.8$ & $6.9 \pm 2.0$ & 0.07 \\
\hline Discharge pain score & $1.9 \pm 2.5$ & $3.4 \pm 2.7$ & 0.002 \\
\hline \multicolumn{4}{|l|}{ Measures of morbidity } \\
\hline Cumulative illness burden (CIRS-G total score) & $17.2 \pm 5.3$ & $19.6 \pm 5.0$ & 0.010 \\
\hline \multicolumn{4}{|c|}{ Multimorbidity severity (number of organ systems with CIRS-G score of $\geq 2$ ) a } \\
\hline Three or more systems & $89(96.7)$ & $49(98.0)$ & 1.00 \\
\hline Four or more systems & $80(87.0)$ & $45(90.0)$ & 0.59 \\
\hline Five or more systems & $59(64.1)$ & $40(80.0)$ & 0.049 \\
\hline
\end{tabular}

Each organ system scored $0-4$ for illness severity: $0=$ none, $1=$ mild, $2=$ moderate, $3=$ severe, $4=$ extremely severe.

${ }^{a}$ All patients in both groups had CIRS-G score of $\geq 2$ in two or more organ systems.

MEDD, morphine equivalent daily dose in milligrams per day.

These findings have a number of key implications. First, older patients are frequently prescribed opioids. Our findings align with several other studies examining opioid use in older adults. Higher rates of opioid prescriptions to older patients with musculoskeletal morbidity, chronic pain, and cancer in our study mirror a large-scale national study that found adults

Table 4. Multivariable Analysis of Factors Associated With OpIOID PRescription at Discharge

\begin{tabular}{lcc}
\hline & \multicolumn{2}{c}{$\begin{array}{c}\text { Discharge opioid } \\
\text { prescription }\end{array}$} \\
\cline { 2 - 3 } Independent variable & $\begin{array}{c}\text { Odds ratio (95\% } \\
\text { confidence interval) }\end{array}$ & $\mathrm{p}$ \\
\hline Age (continuous) & $0.98(0.95-1.01)$ & 0.23 \\
Female & $1.28(0.73-2.23)$ & 0.39 \\
Race (White vs. non-White) & $1.25(0.71-2.18)$ & 0.44 \\
Chronic pain & $2.59(1.45-4.61)$ & 0.001 \\
Cumulative illness burden & $1.08(1.03-1.14)$ & 0.003 \\
$\quad$ & & \\
\hline
\end{tabular}

Adjusted for age, gender, and race. with primary discharge diagnoses of musculoskeletal injury, pain, and cancer were significantly more likely to receive opioids during their hospitalization. ${ }^{31}$ Recent studies of opioid prescribing in hospitalized older adults ${ }^{16}$ and Medicare beneficiaries $^{32}$ also found associations between opioid prescribing and musculoskeletal morbidity. Second, our findings demonstrated overall high illness burden in these hospitalized older adults. Third, the presence of multimorbidity increased the risk of opioid prescribing. Given that multimorbidity also increases risk for opioid-related adverse events, ${ }^{14-16}$ higher illness burden may inform the relationship between opioids and adverse outcomes during and posthospitalization. Maiti et al. ${ }^{16}$ also found that older adults exposed to opioids before and during hospitalization had higher rates of poor outcomes (e.g., longer hospital stay, discharge to skilled nursing facility, and 30-day readmission) than nonexposed older adults. ${ }^{16}$ Maiti and coauthors ${ }^{16}$ did not find a difference in multimorbidity (assessed through the Charlson Comorbidity Index $[\mathrm{CCI}]^{33}$ ) between older medical inpatients exposed and not exposed to opioids. This discrepancy could be explained by differences in study population, differences in the time points of our opioid measures, and by data element differences between the CIRS-G and CCI. However, the discrepancy may 
reflect the importance of comorbidity severity in this relationship, which the CIRS-G captures more fully than the CCI. $^{25,34}$

The association between opioid prescriptions and higher illness burden has several potential explanations. Prescribing physicians may be concerned about the safety of nonopioid analgesics in older patients, particularly those with certain comorbidities such as renal and cardiovascular impairment or those at increased risk for gastrointestinal bleeding. ${ }^{12,35}$ For example, some adjuvant analgesics have anticholinergic effects, which can lead to falls or other adverse cognitive and physical outcomes in elderly. ${ }^{12}$ Opioids may be perceived as safer for those with liver disease (in the case of acetaminophen), renal disease, or a history of gastrointestinal bleeding (in the case of nonsteroidal anti-inflammatory agents). We found that patients who received opioid prescriptions, however, were also more likely to be prescribed an array of analgesics orders for acetaminophen and adjuvant analgesics.

Little evidence exists to guide clinical decision making about opioid prescribing to older adults with pain. Systematic reviews of opioid efficacy and safety have found little data ${ }^{36}$ and low-quality evidence ${ }^{37}$ on outcomes in older adults, and have rarely compared opioids with other analgesics. Studies about the role of opioids with falls and fractures in older adults have reported conflicting results. ${ }^{38-42}$ Furthermore, underuse of opioids may carry risk for older adults with acute pain. In a study of delirium predictors in older adults after hip fracture, delirium incidence increased as opioid dose decreased (from $>30$ to $10-30$ to $<10 \mathrm{mg}$ MEDD). ${ }^{29}$

Evidence for optimal analgesic therapies for older adults is even more limited in the context of multimorbidity. A 2010 meta-analysis of opioid treatment outcomes in older adults found only six studies reporting results on participants aged $\geq 65$ years, and these excluded patients with "significant comorbidity." 43 Furthermore, studies of opioid therapy in older adults with specific comorbidities (e.g., dementia) have focused on postoperative pain, rather than acute or chronic pain. $^{29,41,44}$

The common co-occurrence of pain and multimorbidity in our cohort and other inpatient and community settings $2,13,45$ indicates that physicians regularly face the question of how to safely and effectively treat pain in older adults with multimorbidity. More evidence is needed to guide practice. Clinical research examining opioid efficacy and safety in such a complex population is likely to be challenging, in part, because of growing restrictions on opioid use. Care models such as the Program of All-Inclusive Care for the Elderly ${ }^{46}$ and homebased primary care ${ }^{47}$ that provide close follow-up of older adults with multimorbidity may be ideal settings for studying analgesic-related health outcomes. Such research will be increasingly important as U.S. population ages and as state and federal governments seek to limit opioid misuse and health care expenditure.

\section{Limitations}

This study has several limitations. First, we present data from a single urban academic medical center. However, given the extensive EMR review required to assess comorbidity severity, a large multicenter study on our topic is unlikely. In addition, morbidity measure accuracy could be affected by underdiagnosis of conditions such as cognitive impairment, or by retention of resolved illnesses in the EMR. We addressed the latter by thoroughly reviewing admission and discharge notes and medications to determine the presence and severity of ongoing illnesses.

\section{Conclusion}

Multimorbidity and high illness burden are common among older medical inpatients with pain. Higher comorbidity severity and illness burden is associated with opioid prescribing, and may contribute to posthospitalization adverse events in older adults given opioids. As the population of older adults with multimorbidity grows, additional studies will be critical to identify safer and more effective pain management strategies.

\section{Acknowledgments}

Dr. Schear wishes to thank Dr. Charlotte Carlson for her thoughtful feedback early in this study.

\section{Funding Information}

This study was supported by Tideswell at the University of California, San Francisco (a program supported in part by the Stephen D. Bechtel Jr. Foundation), and by the Medical Student Training in Aging Research Program, sponsored by the National Institute on Aging and American Federation for Aging Research (Grant No. T35AG026736, PI: Jonathan Wanagat). The sponsors had no role in development of the study, data acquisition, data interpretation, writing, or editing of the article.

\section{Author Disclosure Statement}

The authors have no conflicts of interest.

\section{References}

1. American Geriatrics Society Expert Panel on the Care of Older Adults with Multimorbidity: Guiding principles for the care of older adults with multimorbidity: An approach for clinicians. J Am Geriatr Soc 2012;60.

2. Salive ME: Multimorbidity in Older Adults. Epidemiol Rev 2013;35:75-83.

3. Boyd CM, Ritchie C: Addressing Multimorbidity in Older Adults. In: Williams B, Chang A, Ahalt C, et al. (eds): Current Diagnosis \& Treatment: Geriatrics, Vol Second. New York: McGraw Hill, 2014, p. 59.

4. Waldman E, Potter JF: A Prospective evaluation of the cumulative illness rating scale. Aging Clin Exp Res 1992;4: 171-178.

5. Salvi F, Miller MD, Grilli A, et al.: A manual of guidelines to score the modified Cumulative Illness Rating Scale and its validation in acute hospitalized elderly patients. J Am Geriatr Soc 2008;56:1926-1931.

6. Miller MD, Paradis CF, Houck PR, et al.: Rating chronic medical illness burden in geropsychiatric practice and research: Application of the Cumulative Illness Rating Scale. Psychiatry Res 1992;41:237-248.

7. Eckerblad J, Theander K, Ekdahl A, et al.: Symptom burden in community-dwelling older people with multimorbidity: A cross-sectional study. BMC Geriatr 2015; 15:1. 
8. Sawyer P, Bodner EV, Ritchie CS, Allman RM: Pain and pain medication use in community-dwelling older adults. Am J Geriatr Pharmacother 2006;4:316-324.

9. Hunt LJ, Covinsky KE, Yaffe K, et al.: Pain in communitydwelling older adults with dementia: Results from the National Health and Aging Trends Study. J Am Geriatr Soc 2015;63:1503-1511.

10. Won A, Lapane K, Gambassi G: Correlates and management of nonmalignant pain in the nursing home. J Am Geriatr Soc 1999;47:936-942.

11. Johannes CB, Le TK, Zhou X, et al.: The prevalence of chronic pain in United States adults: Results of an Internetbased survey. J Pain 2010;11:1230-1239.

12. American Geriatrics Society Panel on the Pharmacological Management of Persistent Pain in Older Adults: Pharmacological management of persistent pain in older persons. J Am Geriatr Soc 2009;57:1331-1346.

13. American Geriatrics Society Panel on Persistent Pain in Older Adults: The management of persistent pain in older persons. J Am Geriatr Soc 2002;50:S205-S224.

14. Kessler ER, Shah M, Gruschkus SK, Raju A: Cost and quality implications of opioid-based postsurgical pain control using administrative claims data from a large health system: Opioid-related adverse events and their impact on clinical and economic outcomes. Pharmacotherapy 2013; 33:383-391.

15. Minkowitz HS, Gruschkus SK, Shah M, Raju A: Adverse drug events among patients receiving postsurgical opioids in a large health system: Risk factors and outcomes. Am J Heal Pharm 2014;71:1556-1565.

16. Maiti S, Sinvani L, Pisano M, et al.: Opiate prescribing in hospitalized older adults: Patterns and outcomes. J Am Geriatr Soc 2018;66:70-75.

17. Steinman MA, Komaiko KDR, Fung KZ, Ritchie CS: Use of opioids and other analgesics by older adults in the United States, 1999-2010. Pain Med 2015;16:319-327.

18. Mosher HJ, Jiang L, Vaughan Sarrazin MS, et al.: Prevalence and characteristics of hospitalized adults on chronic opioid therapy. J Hosp Med 2014;9:82-87.

19. Dowell D, Haegerich TM, Chou R: CDC Guideline for prescribing opioids for chronic pain-United States, 2016. Morb Mortal Wkly Rep 2016;65:1-50.

20. Deng LX, Patel K, Miaskowski C, et al.: Prevalence and characteristics of hospitalized older adults with moderate to severe pain. J Am Geriatr Soc 2018;66: 1744-1751.

21. Feldt KS: The checklist of nonverbal pain indicators (CNPI). Pain Manag Nurs 2000;1:13-21.

22. Herr KA, Garand L: Assessment and measurement of pain in older adults. Clin Geriatr Med 2001;17:457-478.

23. Jensen M, Karoly P, McCaffery M: Self-report scales and procedures for assessing pain in adults. In: Turk D, Melzack R (eds). Handbook of Pain Assessment. New York: Guilford Press, 2001, pp. 15-34.

24. Edelen M, Saliba D: Correspondence of verbal descriptor and numeric rating scales for pain intensity: An item response theory calibration. J Gerontol A Biol Sci Med Sci 2010;65:778-785.

25. De Groot V, Beckerman H, Lankhorst GJ, Bouter LM: How to measure comorbidity: A critical review of available methods. J Clin Epidemiol 2003;56:221-229.

26. Miller MD, Towers A: A Manual of Guidelines for Scoring the Cumulative Illness Rating Scale for Geriatrics (CIRS-G). Pittsburgh, PA: University of Pittsburgh. 1991;(May):2-31.
27. Borson S, Scanlan JM, Lessig M, DeMers S: Comorbidity in aging and dementia: Scales differ, and the difference matters. Am J Geriatr Psychiatry 2010;18: 999-1006.

28. Valderas JM, Starfield B, Sibbald B, et al.: Defining comorbidity: Implications for understanding health and health services. Ann Fam Med 2009;7:357-363.

29. Morrison RS, Magaziner J, Gilbert M, et al.: Relationship between pain and opioid analgesics on the development of delirium following hip fracture. J Gerontol A Biol Sci Med Sci 2003;58:76-81.

30. Murnion BP, Gnjidic D, Hilmer SN: Prescription and administration of opioids to hospital in-patients, and barriers to effective use. Pain Med 2010;11:58-66.

31. Herzig SJ, Rothberg MB, Cheung M, et al.: Opioid utilization and opioid-related adverse events in nonsurgical patients in US hospitals. J Hosp Med 2014;9:73-81.

32. Jena AB, Goldman D, Karaca-Mandic P: Hospital prescribing of opioids to medicare beneficiaries. JAMA Intern Med 2016;02115:990-997.

33. Charlson ME, Pompei P, Ales KL, MacKenzie R: A new method of classifying prognostic in longitudinal studies: Development and validation. J Chronic Dis 1987;40:373383.

34. Rochon PA, Katz JN, Morrow LA, et al.: Comorbid illness is associated with survival and length of hospital stay in patients with chronic disability: A prospective comparison of three comorbidity indices. Med Care 1996;34:10931101.

35. Gabriel SE, Jaakkimainen L, Bombardier C: Risk for serious gastrointestinal complications related to use of nonsteroidal anti-inflammatory drugs: A meta-analysis. Ann Intern Med 1991;115:787-796.

36. Chou R, Turner JA, Devine EB, et al.: The effectiveness and risks of long-term opioid therapy for chronic pain: A systematic review for a national institutes of health pathways to prevention workshop. Ann Intern Med 2015;162: 276-286.

37. Megale RZ, Deveza LA, Blyth FM, et al.: Efficacy and safety of oral and transdermal opioid analgesics for musculoskeletal pain in older adults: A systematic review of randomized, placebo-controlled trials. J Pain 2018;19: 475.e1-e475.e24.

38. Krebs EE, Paudel M, Taylor BC, et al.: Association of opioids with falls, fractures, and physical performance among older men with persistent musculoskeletal pain. J Gen Intern Med 2016;31:463-469.

39. Miller M, Stürmer T, Azrael D, et al.: Opioid analgesics and the risk of fractures in older adults with arthritis. J Am Geriatr Soc 2011;59:430-438.

40. Lo-Ciganic WH, Floden L, Lee JK, et al.: Analgesic use and risk of recurrent falls in participants with or at risk of knee osteoarthritis: Data from the osteoarthritis initiative. Osteoarthritis Cartilage 2017;25:1390-1398.

41. Vaurio LE, Sands LP, Wang Y, et al.: Postoperative delirium: The importance of pain and pain management. Anesth Analg 2006;102:1267-1273.

42. Rolita L, Spegman A, Tang X, Cronstein BN: Increasing narcotic analgesic prescriptions for osteoarthritis is associated with increased falls and fractures in the elderly. J Am Geriatr Soc 2013;61:335-340.

43. Papaleontiou M, Henderson CR Jr., Turner BJ, et al.: Outcomes associated with opioid use in the treatment of chronic non-cancer pain among older adults: A systematic 
review and meta-analysis. J Am Geriatr Soc 2010;58:13531369.

44. Sieber FE, Mears S, Lee H, Gottschalk A: Postoperative opioid consumption and its relationship to cognitive function in older adults with hip fracture. J Am Geriatr Soc 2011;59:2256-2262.

45. Fortin M, Bravo G, Hudon C, et al.: Prevalence of multimorbidity among adults seen in family practice. Ann Fam Med 2005;3:223-228.

46. Eng C, Pedulla J, Eleazer P, et al.: Program of All-Inclusive Care For The Elderly (PACE): An innovative model of integrated geriatric care and financing. J Am Geriatr Soc 1997;45:223-232.
47. Stall N, Nowaczynski M, Sinha SK: Systematic review of outcomes from home-based primary care programs for homebound older adults. J Am Geriatr Soc 2014;62:2243-2251.

Address correspondence to: Christine S. Ritchie, MD, MSPH Division of Geriatrics Department of Medicine University of California San Francisco 3333 California Street, Suite 380 San Francisco, CA 94143-1265

E-mail: christine.ritchie@ucsf.edu 